\title{
A Hysteresis-Based Steering Feel Model for Steer-by-Wire Systems
}

\author{
M. Selçuk Arslan \\ Department of Mechatronics Engineering, Yildiz Technical University, Istanbul, Turkey \\ Correspondence should be addressed to M. Selçuk Arslan; msarslan@yildiz.edu.tr
}

Received 1 March 2017; Accepted 18 September 2017; Published 1 November 2017

Academic Editor: Mohammed Nouari

Copyright (C) 2017 M. Selçuk Arslan. This is an open access article distributed under the Creative Commons Attribution License, which permits unrestricted use, distribution, and reproduction in any medium, provided the original work is properly cited.

\begin{abstract}
A mathematical model of steering feel based on a hysteresis model is proposed for Steer-by-Wire systems. The normalized BoucWen hysteresis model is used to describe the steering wheel torque feedback to the driver. By modifying the mathematical model of the hysteresis model for a steering system and adding custom parameters, the availability of adjusting the shape of steering feel model for various physical and dynamic conditions increases. Addition of a term about the tire dynamics to the steering feel model renders the steering wheel torque feedback more informative about the tire road interaction. Some simulation results are presented to establish the feasibility of the proposed model. The results of hardware-in-the-loop simulations show that the model provides a realistic and informative steering feel.
\end{abstract}

\section{Introduction}

In road vehicles, the steering action is performed through the mechanical link between the wheels and the steering wheel. When the road wheels are steered, a reaction torque, which is mainly based on the self-aligning torque (SAT), occurs at the steering wheel, and the driver experiences the steering feel. Since the steering feel has a significant effect on the handling quality by providing the driver with accurate control of the vehicle, it is recognized as an essential feedback to the driver [1]. Due to many factors-such as the external forces acting on the driver in maneuvering a vehicle, the interaction between the driver's arm and the steering wheel, and other sensory information to the driver-the effect of torque feedback on the driver is not understood well. Although there are studies in the literature on objective and subjective evaluations of the steering feel [2-8], they are still in their infancy.

The steering technology after active front steering appears to be the Steer-by-Wire (SBW) technology for the road vehicles. Researches on SBW are mainly conducted in the fields of active steering control and steering feel [9-13]. In SBW systems, elimination of the steering column results in the loss of connection between the front road wheels and steering wheel. A force-feedback system becomes indispensable for providing a steering feel to the driver. The need for generating a virtual steering feel has substantially drawn the attention along with the studies on the control of SBW systems. In some researches, conventional steering feel was studied and promising results were obtained $[14,15]$. In order to calculate the steering wheel motor torque, a torque map, which depends on the steering wheel angle and vehicle speed, was introduced by Kim et al. [16]. Some studies report the use of road wheel reaction torque by measuring the forces at road wheel (rack force) real-time to provide a torque feedback to the driver $[17,18]$. A few researches have proposed some force-feedback models which use information from vehicle dynamics save for the self-aligning torque [19]. Particularly, attention is given to the use of lateral acceleration and yaw rate in the design of steering feel model. These variables appear as highly related to the steering, though how they can be used in designing the steering feel is not clear.

Most of the studies in the literature mainly or partially use estimated SAT information to generate the conventional steering feel [20-23]. Estimation of SAT brings some difficulties and complexity. Also, it is not suitable for lower velocities and when the vehicle is stationary. An additional approach seems to be needed to reproduce the steering feel in such 
cases, when the model based on the SAT is not applicable. On the other hand, it is important to note that using SAT in developing steering feel model would cause a problem in the steering maneuver of the driver, while an active steering control is in operation. Simply, use of the self-aligning torque means the steering motion of the road wheels is reflected to the driver. The interference of corrective control command of the active steering control results in a different steering motion of the road wheels apart from the driver's steering command. Feeding the SAT back to the driver during steering would have a disturbing effect.

The steering wheel torque had to be designed so that the vehicle dynamics influencing the steering wheel torque reflects the maneuvering dynamics correctly. In the design of steering feel, the reflection of road wheels and road interaction to the driver is of crucial importance. In provision of this feedback, the steering wheel torque in the steering wheel system can be generated based on either the force signal from load cells attached to the rack or the mathematical model of steering wheel torque which is indispensably dependent on the estimation of tire parameters. When the cost, replacement, and calibrations of the load cell are taken into account, it is understood that the estimation based model would be more suitable.

It can be said, based on the studies of steering feel in the literature [24], that the most important and main characteristic influencing the steering feel is evident in the relation between steering wheel angle and steering wheel torque feedback. In real driving tests, this relation was identified as a curve resembling to the Lissajous curve [25] and used by Morita et al. [26] as a criterion or mostly described as a hysteresis curve.

As stated by Balachandran and Gerdes [23], the steering feel model is designed so that it must be both complex enough to capture all elements of steering feel and simple enough to be tuned intuitively. There is still no general and sufficient model for designing the steering feel in the literature. We understand that the model defining the steering feel should be characterized by not only objective measures but also subjective measures obtained by the results of driving tests done by human drivers. In the search of a flexible model with appropriate fidelity, hysteresis-based model has arose. In this respect, it is necessary to emphasize that since the subjective evaluation of steering response is not within the scope of this study, the steering feel actually refers to the artificial steering feedback in this work.

The purpose of using hysteresis-based model is twofold. First, the elimination of mechanical connection in the steering system poses an essential question: what would be the form and magnitude of steering wheel torque compared to the one in conventional vehicles? There is no requirement or evidence that they both must be the same. In the search of answer, the hysteresis-based model would be a useful tool by providing the flexibility and the ease of design and tuning. Second, the hysteresis-based model can pattern the hysteresis characteristic appearing in the conventional steering systems.

The experimental studies in the literature show that a lag characterizes the relation between the steering wheel angle and the steering wheel torque feedback. It is well-known that the vehicle dynamics is dominated by tire dynamics. Mainly the relaxation length in a tire is responsible for the lag, which delays the tire side forces $[27,28]$. The behavior of hysteresis curve is also characterized by a lag between input and output. In this context, the Bouc-Wen hysteresis model has been selected to model the steering feel [29]. Its mathematical simplicity and suitability for modeling the mechanical systems have been the factors in selecting the Bouc-Wen model.

The Bouc-Wen hysteresis model itself cannot describe the steering dynamics of a vehicle fully. The effect of speed on steering feel is one of the parameters influencing the steering dynamics. At higher speeds, the vehicle may not be controlled for higher steering angles. The torque feedback felt by driver at high speeds restricts the driver from turning the steering wheel excessively. In the same way, it is expected that the driver makes less effort to turn the steering wheel for larger angles at lower speeds. By making the shape of hysteresis speed dependent, namely, relating the gradient of the hysteresis curve with the longitudinal velocity, the steering wheel torque has been generated at the correct level. Another aspect of the steering dynamics is the interaction between tire and road. The variation in the lateral tire force is valuable feedback to the driver. The hysteresis model itself does not hold dynamics at the contact patch level. To keep the driver informed about the lateral dynamics of front tires, a term has been added to the hysteresis model. This term holds the information in the variation of lateral tire force. The pneumatic trail of a tire presents rich information in this respect, and it can be obtained through estimation. By using the pneumatic trail in this term, the reduction in the SAT due to the saturation of lateral tire force can be reflected to the driver.

To test the performance of the proposed steering feel model system, the hardware-in-the-loop (HIL) simulation approach has been used. The hardware-under-test emulates the steering system of a conventional passenger car. The software part consists of a 10-DOF full vehicle model and the target control system. To perform simulations, two case studies based on standard test procedures have been presented: the weave test and double lane-change test. The performance of a vehicle with hysteresis-based steering feel model has been compared with the same vehicle with SAT-based model. The reason of selecting the SAT-based model is to compare the proposed steering feel model with the steering feel generated in conventional vehicles. In this way, a realistic comparison could be done.

The outline of this work is as follows: In Section 2, the Bouc-Wen hysteresis model is introduced and the steering feel model based on this hysteresis model is proposed. The HIL simulation environment is introduced in Section 3. The standard test procedures are described and the results of case studies are given in Section 4.

\section{Steering Feel Design}

In conventional steering systems, the motion of steering wheel is transmitted to the road wheels mechanically. The resulting rotation of road wheel in a vehicle with longitudinal 
velocity causes the generation of lateral force acting on the wheel. The lateral force with respect to swivel axis creates the self-aligning moment. Another tire moment, the jacking torque, which causes self-centering effect at low speeds, occurs due to the effect of normal forces in the rotated wheels. Additionally, the effect of inertia and damping in the steering system contributes to the total torque felt in the steering wheel together with aforementioned moments.

In SBW systems, the rotational motion of the steering wheel is processed by an electronic control unit and transferred to the steering actuator attached to the rack. In theory, the desired steering feel for an SBW system can be reproduced independently of the static and dynamic effects of steering system in a conventional vehicle. However, by decoupling the steering wheel from road wheels, the reaction torque occurred in road wheels, which directly affects the neuromuscular system of a driver through steering wheel, the human driver becomes mechanically isolated from the dynamic interaction of road wheels and road. The loss of such very direct feeling deprives the driver of an important feedback.

The steering wheel torque experienced by a driver is an important piece of information, since this feedback signal facilitates the safety and quality of driving significantly [1]. Providing the steering feedback to the driver has an effect on improving the driver's ability to evaluate the state of vehicle. Thus, the driver learns and stores the knowledge of nonlinear vehicle dynamics.

2.1. Bouc-Wen Model of Hysteresis. One of the nonlinear behaviors usually encountered in physical systems is the hysteresis. This behavior in terms of input-output relation is characterized by memory. The output is formed depending upon the history of the input signal in the systems with memory. For instance, hysteresis characterizes the restoring force that materials generate against the movement in mechanical, aerodynamic, and structural systems. In those systems, the restoring force is dependent on not only the instantaneous deformation but also the history of deformation [30].

Hysteresis encountered in many applications, including magnetic, superconducting, optical, adsorption, economic, and mechanical applications. In modeling the hysteretic processes, one of the widely known models to describe the behavior of ferromagnetic materials was suggested by Preisach, as geometric interpretation of the model proposed by Weiss and Freudenreich [31]. The other well-known models of hysteresis were studied by Duhem [31], Ishlinskii [31], Chua and Stromsmoe [32], and Jiles and Atherton [33]. The reader can refer to the work of Mayergoyz for other mathematical models for hysteresis [34]. From among those models, the Bouc-Wen model or its derivatives can be used to model hysterical systems, especially for modeling the structures, magnetorheological dampers, base isolation systems, and various dampers [35]. Bouc-Wen model is adopted in this study to model the steering wheel torque.

Bouc-Wen model of hysteresis is introduced by Bouc and generalized by Wen [36, 37]. This model describes the output restoring force to the input displacement in the form of a first-order nonlinear differential equation. The nonlinear hysteretic behavior of a physical system can be described as a map $x(t) \mapsto \Phi(t)$, where $x$ and $\Phi(x)$ represent the time histories of an input and hysteretic output, respectively. It is known that, for any bounded input $x(t)$, the output $\Phi(x)(t)$ is bounded [35]. The hysteretic behavior can be described by the Bouc-Wen model:

$$
\begin{aligned}
\Phi(x)(t) & =\alpha k x(t)+(1-\alpha) D k z(t), \\
\dot{z} & =D^{-1}\left(A \dot{x}-\beta|\dot{x}||z|^{n-1} z-\gamma \dot{x}|z|^{n}\right),
\end{aligned}
$$

where $A, B, D, n, \alpha, \beta$, and $\gamma$ are the parameters controlling the shape of hysteresis loop. It is apparent that there are seven parameters to be determined in this model. Another model exactly equivalent to this standard model is preferred in this study, since it needs the identification of less parameters. By defining

$$
\omega(t)=\frac{z(t)}{z_{0}},
$$

the model can be written as

$$
\begin{aligned}
& \Phi(x)(t)=\kappa_{x} x(t)+\kappa_{\omega} \omega(t), \\
& \dot{\omega}(t)=\rho\left(\dot{x}(t)-\sigma|\dot{x}(t)||\omega(t)|^{n-1} \omega(t)\right. \\
& \left.\quad+(\sigma-1) \dot{x}(t)|\omega(t)|^{n}\right),
\end{aligned}
$$

with an initial condition $\omega(0)$, where

$$
\begin{aligned}
\rho & =\frac{A}{D z_{0}}>0, \\
\sigma & =\frac{\beta}{\beta+\gamma} \geq 0, \\
\kappa_{x} & =k \alpha>0, \\
\kappa_{\omega} & =(1-\alpha) D k z_{0}>0 .
\end{aligned}
$$

Equations (3a) and (3b) are known as normalized form of Bouc-Wen model. If the initial condition $\omega(t)$ is guaranteed such that $|\omega(0)| \leq 1$, then, $|\omega(t)| \leq 1$ for all $t \geq 0$ [35]. The parameters are all unknown and can be set to obtain the desired shape of hysteresis. The displacement, $x(t)$, and the velocity, $\dot{x}(t)$, can be acquired from sensors.

Some mechanical systems with hysteresis can be represented by a mass-damper system and a hysteresis element as shown in Figure 1. Such mechanical system holds the dynamics of the steering system studied in this research.

The second-order mechanical model for such a system can be defined as follows:

$$
m \ddot{x}+c \dot{x}+\Phi(x)(t)=F
$$

with initial conditions $x(0)$ and $\dot{x}(0)$. The system is excited by an input force, $F$. The restoring force, $\Phi(x)(t)$, is assumed to be described by the normalized Bouc-Wen model given in (3a) and (3b). This mathematical model forms the basis for this study. 


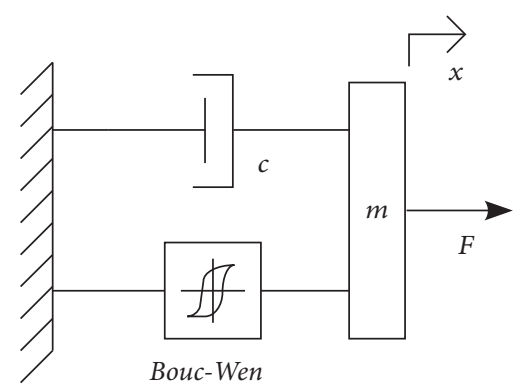

Figure 1: Mass-damper system with hysteresis element.

The input-output hysteretic behavior of normalized Bouc-Wen model is shown in Figure 2. A sinusoidal signal with increasing amplitude from 0 to 1 has been employed as input in (3a) and (3b). As can be seen from Figure 2(b), after the transient behavior, limit cycle of the hysteresis occurs. Since it is a normalized model, the output stays between -1 and 1 . Here, the parameters are arbitrarily selected as $\kappa_{x}=$ $0.01, \kappa_{\omega}=1, \rho=2, \sigma=0.05$, and $n=2$.

In order to determine the role of parameters on controlling the shape of the hysteresis loop, the one-factor-at-a-time method is applied. In this method, one parameter is varied at a time while the others are kept fixed at preselected values. Even though this method gives us only local results, it is still quite useful in understanding the control of the hysteresis loop. The values of parameters given previously are taken as nominal values. By varying one parameter at a time in (3a) and (3b), for the same sinusoidal signal input, the restoring force outputs have been plotted against the displacement as shown in Figures 3(b)-3(f).

To evaluate the results given in Figures 3(b)-3(f), we can define the properties of a hysteresis loop given in Figure 3(a) as (i) the abscissa deadband as the distance between the upper and lower points where the curve crosses the vertical line, (ii) the ordinate deadband as the distance between the leftmost and rightmost points where the curve crosses the horizontal line, and (iii) the hysteresis gradient as the ratio of change in restoring force to change in displacement. When the input is the steering wheel angle and the output is the steering wheel torque, the hysteresis gradient implies the steering stiffness. The parameter $\kappa_{x}$ has an effect mainly on the hysteresis gradient. The extremum points of the hysteresis loop are also affected by the change of parameters in (3a), since $\Phi(x)(t)$ is proportional to the value of $\kappa_{x}$, as well as to the value of $\kappa_{\omega}$. The main effect of $\kappa_{\omega}$ is on the abscissa deadband. As shown in Figure 3(d), $\rho$ is responsible for the ductility of the shape and controls the hysteresis gradient without affecting on the extremum points of the loop. By increasing $\rho$, the maximum value of the hysteretic output reaches to unity asymptotically. Another parameter responsible for the hardening and the softening of the shape is $\sigma$. It also controls the ordinate deadband. The last parameter $n$ influences the transition from linear to plastic behavior.

As a side note, among the parameters controlling the shape of the hysteresis, $n$ is the least sensitive and $\kappa_{\omega}$ is the most sensitive to parameter changes [38]. For more detailed and analytical studies on the parameter variations of the Bouc-Wen hysteresis model, the reader can refer to the work of Ikhouane and Rodellar [35].

2.2. Mathematical Model of the Steering Feel. The model of the steering system can be described as a nonlinear system, whose inputs are the steering wheel angle, $\theta$, the longitudinal velocity of the vehicle, $u$, and the pneumatic trail, $\xi$, and whose output is the steering wheel torque, $T_{s w}$, generated by an electric motor. The illustration depicting the signal flow between components of the system is given in Figure 4. The details of the physical setup and vehicle dynamics model are described in Section 3. In Figure 4, steering wheel system represents the physical system with angular position signal, which is $\theta$. While $\theta$ is an input to the mathematical model of vehicle, it is fed to the steering feel model at the same time. Another input employed in the steering feel model is the longitudinal velocity of the vehicle, $u$. The third input, $\xi$, is used to reflect the tire road interaction to the steering feel model. The Bouc-Wen hysteresis model introduced in the previous section is used as the steering feel model to produce the unknown restoring force.

The steering system can be modeled as a single degree-offreedom system

$$
J_{\mathrm{sw}} \ddot{\theta}+b \dot{\theta}+T_{\mathrm{sw}}=T_{d}
$$

where $J_{\text {sw }}$ and $b$ are the system inertia and the damping coefficient, respectively. The angular position of the steering wheel is represented by $\theta$, while $T_{\mathrm{sw}}$ denotes the commanded steering reaction torque characterizing the hysteretic behavior of the steering. The torque applied to the driver, $T_{d}$, appears as the combined effects of inertia, damping, and the steering reaction torque on the left-hand side of (6).

2.2.1. Speed Dependency of the Model. In driving a vehicle, it is known that the range of steering angle used by the driver is greater in lower speeds than that of higher speeds. In general, the torque feedback at higher speeds is used by the driver to control the vehicle, whereas the steering angle is used at lower speeds. Based on this fact, the relation between the steering angle and the feedback torque must not be considered as linear. The variation of SAT for different longitudinal velocities, where the same sinusoidal steering input is applied to the vehicle, is shown in Figure 5(a). Note that the values of torques, forces, and displacements are normalized to one in these figures. As can be seen from the figure, the gradient and ductility of the hysteresis loops vary depending on the speed. It is clear that, as in power assisted steering systems, the speed must be employed in the generation of steering feel. For this purpose, the parameter $\rho$ is designed as a function of longitudinal velocity, $u$. The effect of function $\rho(u)$ on the shape of hysteresis loop in different speeds, for the same parameters aforementioned, can be seen in Figure 5(b).

The function $\rho(u)$ is determined depending on the dynamic characteristics of a vehicle. In this study, a linear function is selected: $\rho(u)=k_{u} u$. Here, $k_{u}$ is a constant coefficient. It is understood from Figure 5(b) that the function 

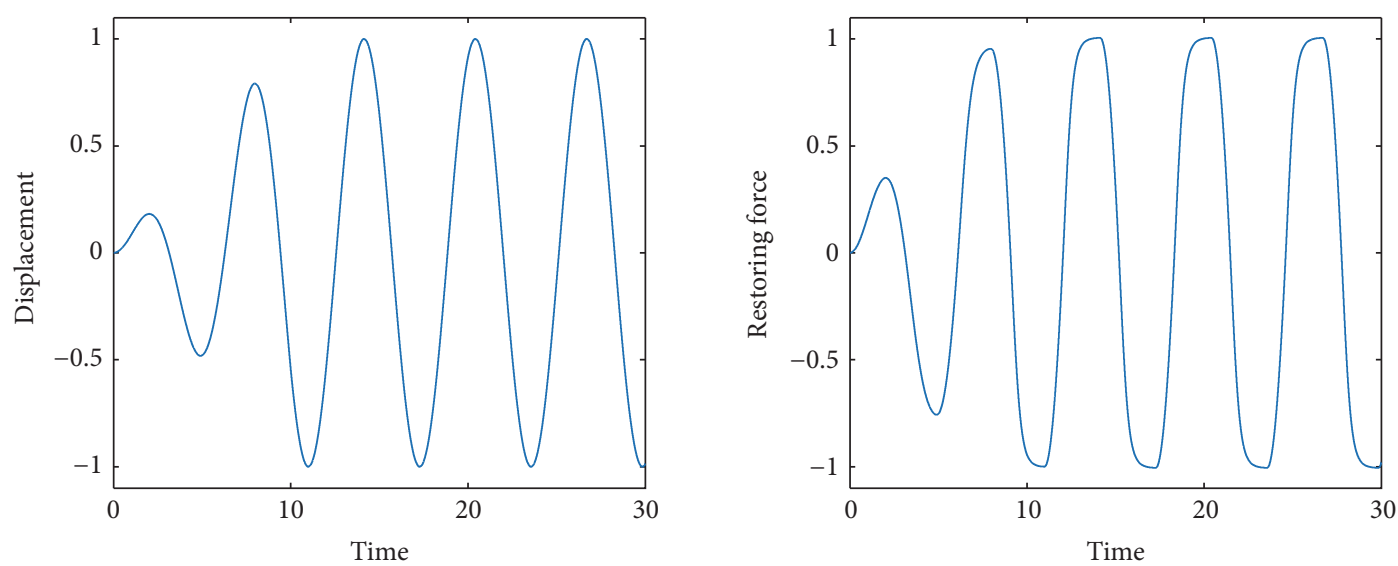

(a)

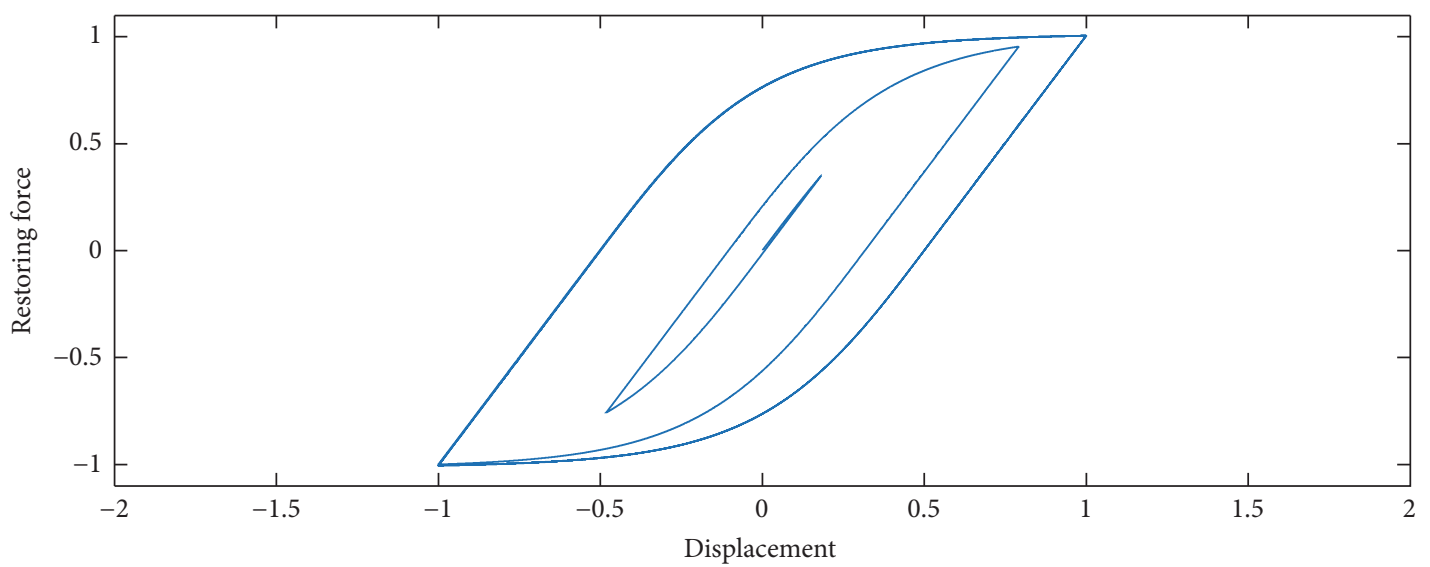

(b)

FIGURE 2: Input-output hysteretic behavior of normalized Bouc-Wen model.

$\rho(u)$ is able to form the shape of hysteresis loop depending on the longitudinal velocity.

\subsubsection{Integration of the Tire Road Interaction. If the repro-} duction of the steering wheel torque based on the steering angle has a big role on the steering feel, it can be said that the feedback torque reflecting the tire road interaction to the driver has a critical role. The SAT plays this critical role and holds information as an indication of approaching the lateral limits of tire. As the tire approaches its limit, the SAT and the steering wheel torque reduce, even if the lateral force increases. In conventional vehicles, such reduction in the steering wheel torque is a crucial feedback and warns the driver of the potential hazard [39]. The decrease after the SAT value reaches its peak value due to tire force saturation can be seen in Figure 5(a) for higher velocities. Such situation may occur not only in higher velocities, but also in lower velocities when the friction force is low as well.

The SAT is generated by the lateral force acting at the pneumatic trail, and both are variable. It is empirically known that the pneumatic trail goes to zero while the SAT is decreasing. We find that the pneumatic trail is a key factor in the dynamic interaction of tire and road. Use of tire lateral force in early determination of the limits for small angles may not be satisfactory. However, the pneumatic trail has potential of enabling the early detection of the tire's handling limits, since it is sensitive to the friction limit even when lateral force is in the linear region of handling [40]. To calculate the pneumatic trail, a tire model [41], or an estimation approach, for example, as discussed by Judy Hsu et al. [40], can be used.

As motivated by the decrease in steering wheel torque when the cornering force saturates in conventional vehicles, the restoring force output defined in (3a) can be altered. Instead of modifying the hysteresis model, the torque representing the decrease is incorporated into the steering wheel torque. Thus, the reduction in steering wheel torque, $T_{r}$, can be defined as proportional to the restoring force and as a function of the pneumatic trail, $\xi$ :

$$
T_{r}=\left(\kappa_{x} x(t)+\kappa_{\omega} \omega(t)\right)(1-\xi) u_{n}
$$

where $u_{n}$ is the normalized longitudinal velocity, and it can be defined as $u_{n}=u / u_{\max }$. By selecting a maximum longitudinal velocity, $u_{\max }$, the effect of decreasing torque can be incorporated into $\Phi(x)(t)$ depending on the longitudinal velocity. The parameter $u_{n}$ can be used to keep the limits of hysteresis near each other, although use of it in (7) may be optional. If (3a) and (7) are arranged, $T_{r}$ can be integrated 

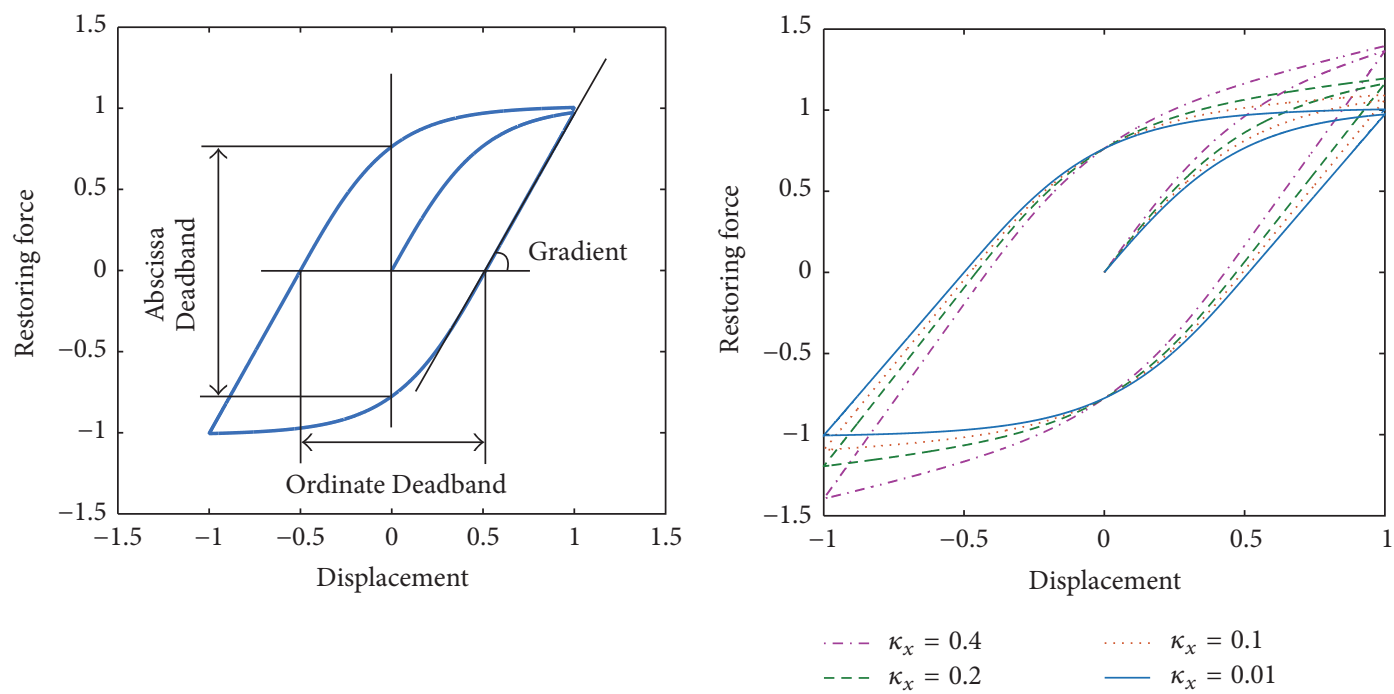

(a)

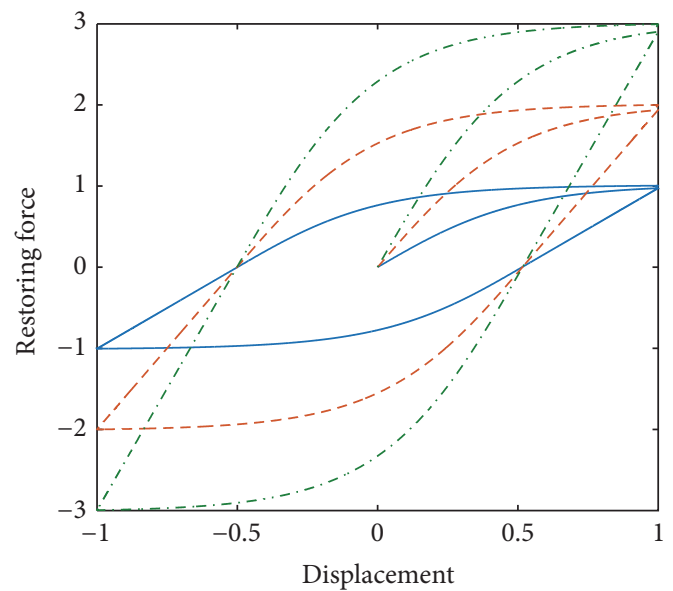

(b)

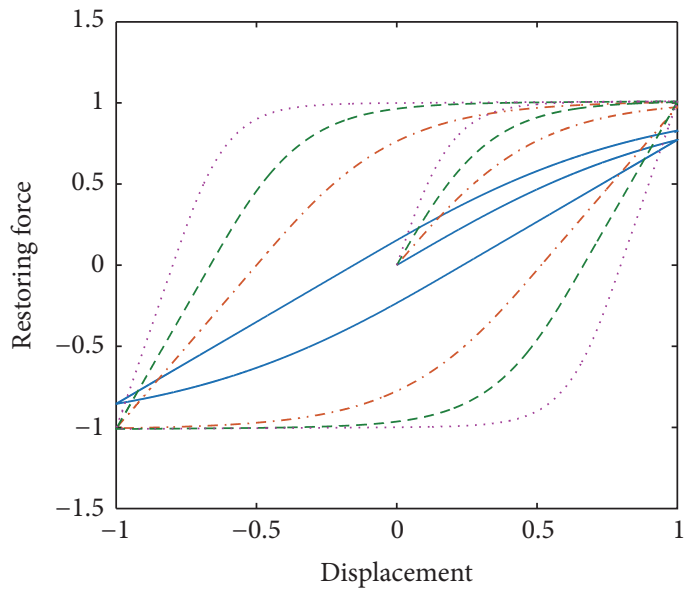

$$
\begin{aligned}
- & \kappa_{\omega}=1 \\
--- & \kappa_{\omega}=2 \\
-\cdot- & \kappa_{\omega}=3
\end{aligned}
$$

(c)

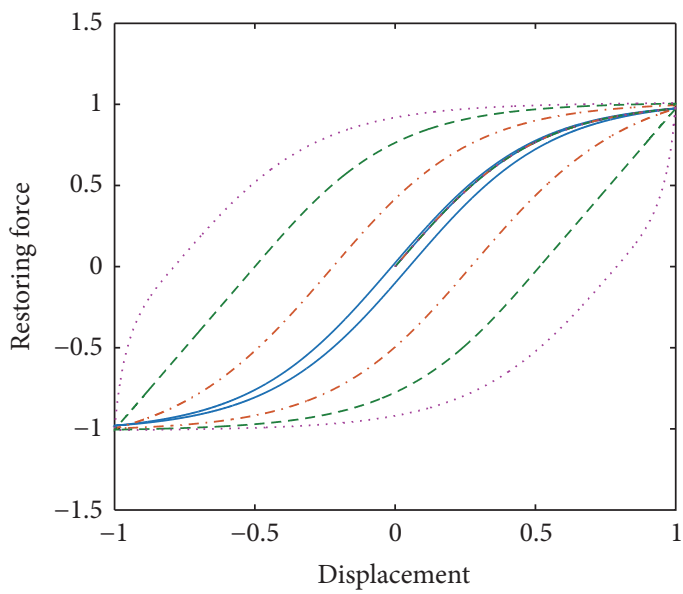

$$
\begin{aligned}
& -\rho=1 \\
& \text {-. } \rho=2 \\
& \text { - - } \rho=3
\end{aligned}
$$

(d)

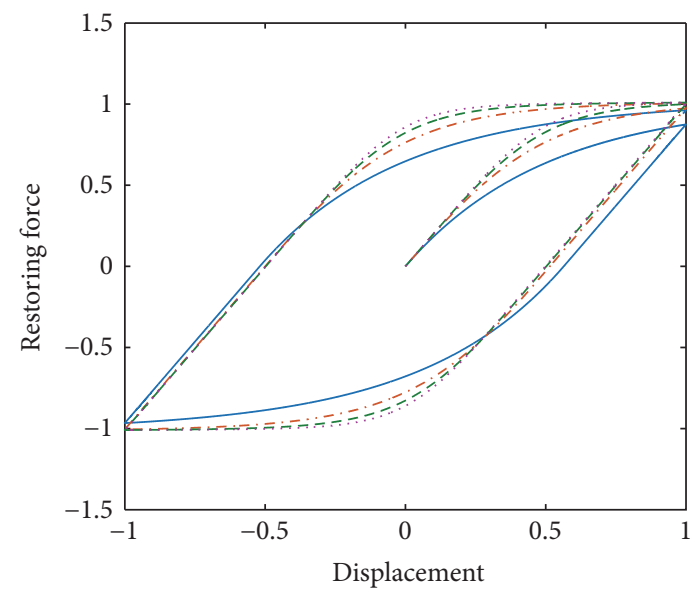

$$
\begin{aligned}
-\sigma & =0.01 & ---\sigma & =0.5 \\
--\sigma & =0.1 & \ldots & \sigma
\end{aligned}
$$

(e)

$$
--n=3
$$$$
n=2
$$

(f)

FIGURE 3: Properties of a hysteresis loop (a), and hysteresis loops by change of (b) $\kappa_{x}$, (c) $\kappa_{\omega}$, (d) $\rho$, (e) $\sigma$, and (f) $n$. 


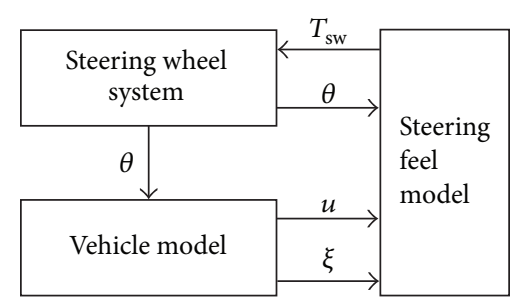

FIGURE 4: Signal flow between components of the steering system.

with the restoring force so as to constitute the steering wheel torque and, thus, (3a) can be rewritten as

$$
\Phi(x)(t)=\kappa_{x} x(t)+\kappa_{\omega} \omega(t)-T_{r} .
$$

The effect of torque reduction is tested for different initial velocities, $u_{0}$, by applying a sinusoidal steering input to the vehicle. The values of parameters are those given in Section 2.1. The results for $\Phi(x)(t)$ with torque reduction based on (8) are given in Figures 6(a)-6(c) together with the plots of $\Phi(x)(t)$ without torque reduction. The variation of $\xi$ in different velocities for the same steering input is seen in Figure 6(d). After some value of $\xi$ is reached, a distinguishable effect of torque reduction can be noted in the figures. Note that the change of SAT for different, especially higher, velocities is given in Figure 5(a). A different form of torque reduction is obtained here compared to the reduction form that appears in SAT. At higher velocities, the effect of $T_{r}$ occurs as a sudden reduction, where a notable change in the kinesthetic sense of a human driver can take place. This kind of change as a feedback to the driver can be more informative about the dynamic condition of tires.

2.2.3. The Final Model. Based on the normalized Bouc-Wen model given in (3a) and (3b), the hysteresis-based steering feel model for a road vehicle can be built by adding the speed dependency and tire road interaction to the model. By defining the normalized steering wheel angle, $\theta_{n}=\theta / \theta_{m}$, where $\theta$ denotes the steering wheel angle and $\theta_{m}$ is the maximum steering wheel angle from the center, the hysteresisbased steering feel model can be introduced as

$$
\begin{aligned}
T_{\mathrm{sW}} & =\kappa_{x} \theta_{n}+\kappa_{\omega} \omega-T_{r}, \\
\dot{\omega} & =\rho(u)\left(\dot{\theta}_{n}-\sigma\left|\dot{\theta}_{n}\right||\omega|^{n-1} \omega+(\sigma-1) \dot{\theta}_{n}|\omega|^{n}\right),
\end{aligned}
$$

where $T_{\mathrm{sw}}$ is the steering wheel torque to be generated by the steering wheel actuator. In (9a), $T_{r}$ can be treated as an optional term, since its significance depends on the generation of side-slip angle. In vehicles with very low speed, such as agricultural vehicles or earth-moving machinery, $T_{r}$ would have a neglected contribution to $T_{\mathrm{sw}}$ due to very small values of side-slip angle.

\section{HIL Simulation System}

An HIL simulation environment has been designed for the steering system with the steering feel model to evaluate the designed mathematical model. In the HIL simulation environment, a hardware-under-test is substituted for its mathematical model in a simulation model of the whole system, and an interface between the physical system and the mathematical model representing rest of the vehicle is designed. In this research, the steering wheel system is the hardware-under-test. The HIL simulation environment is introduced here without entering into details.

For HIL simulations, a driving simulator with a steering wheel system has been built as shown in Figure 7(a). The physical system, steering feel model, and mathematical model of the vehicle all were interfaced with each other in a closedloop by means of a controller board. The steering system mainly consists of the steering wheel and an electric motor attached to the steering wheel by a flanged connector. The $\mathrm{NSK}^{\mathrm{TM}}$ Megatorque direct drive motor, which is an AC servo motor with $12 \mathrm{Nm}$ maximum torque, is used as the actuator. In this study, the servo motor is controlled in the torque mode by sending the control signal generated as a voltage from designed controller to the motor driver through the controller board.

The embedded software part mainly consists of the steering feel model and the vehicle model. The vehicle model and the steering feel model were designed in Matlab ${ }^{\mathrm{TM}}$, Simulink, and the I/O connections with the controller board were made through Simulink. The built code of simulation model has been embedded to the dSPACE ${ }^{\mathrm{TM}}$ DS1103 rapid controller prototyping board.

In order to have realistic results, a detailed nonlinear model of the vehicle has been used in the simulations. The mathematical model is a 10-DOF full vehicle model, which consists of the chassis and suspension dynamics. The vehicle body has 6-DOF, and each of four wheels has 1-DOF. The vehicle is a front wheel steered vehicle. It is accepted that the front and right wheels have the same steering angle, which is 1-DOF, and this DOF is not included in the model since it is represented by the physical system. The mathematical model of vehicle is not given here, but the reader is referred to the works of Abe and Kiencke and Nielsen for the mathematical models used in this study $[41,42]$.

The well-known brush tire model [43] is used to model the tires. The model used for the self-aligning torque generated in a tire is also based on the brush tire model. In calculating the SAT, the effect of steering kinematics due to the kingpin inclination, the caster angle, and the camber angle is ignored. The friction coefficient between road and tire is calculated by a function of side-slip velocity for a dry road condition. The vehicle model takes into consideration the vertical load change for each wheel separately due to roll and pitch motions of the body.

The reader is referred to the work of [9] for the validation of the same vehicle model used in this study. As indicated in that work, the validation study showed that the vehicle model is appropriate for performing HIL simulations accurately.

The steering feel model described in Section 2 was employed in the driving simulator. The steering angle is directly fed from the encoder of servo motor to the models of steering feel and vehicle. The steering feedback torque calculated by (9a) and (9b) is converted to the voltage signal 


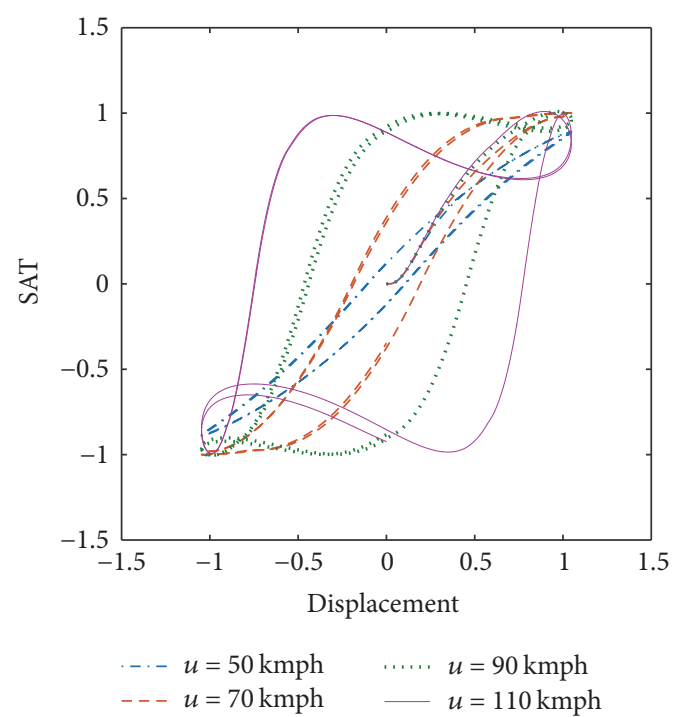

(a)

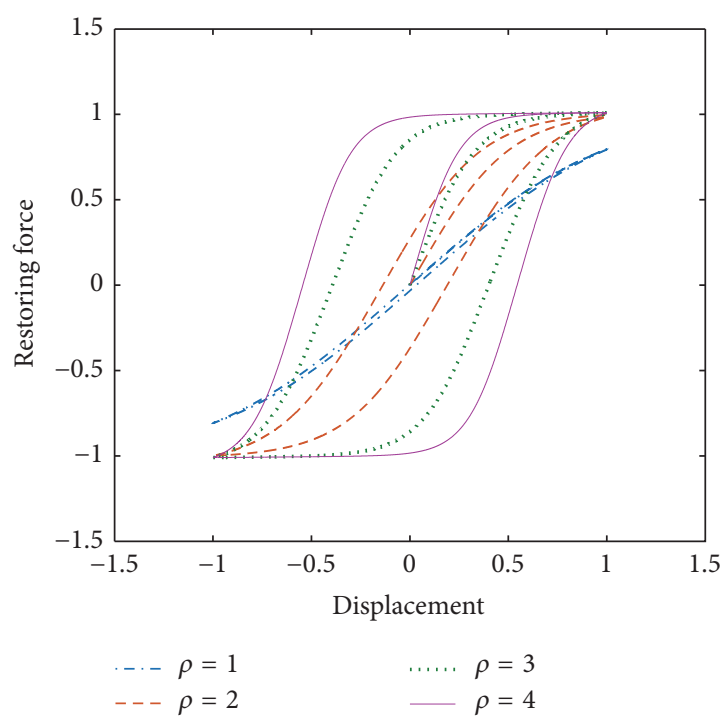

(b)

FIGURE 5: Variation of the (a) SAT and (b) restoring force with respect to speed.

and sent to the servo motor driver. General architecture of the HIL simulation environment is shown in Figure 7(b). The graphical environment has been designed by using the software MotionDesk by dSPACE. The virtual reality part of the simulation has been carried out by a high performance computer, which provides a high quality visual feedback. Also, any time delay in the motion of vehicle in the graphical environment in response to the input of driver has not been experienced.

The return-to-center response of steering wheel from an off-center release affects the handling of a vehicle. The design of a position controller for obtaining a desired return-tocenter response, which is particularly characterized by the damping behavior of the steering wheel, is not included in this study. An additional position controller can be considered for the proposed system for a desired response, although the steering wheel returns to the center satisfactorily in the steering system with hysteresis-based steering feel model, thanks to the current torque control.

\section{Test Results}

The proposed steering feel model has been tested in the test setup and compared with a model based on SAT. The SATbased model is simply obtained by multiplying a predetermined gain by the SAT signal, which is calculated through the formula given in the work of Abe [41]. Tests have been done for two case studies: Weave test and double lane-change test. In the tests, the driver, who has almost twenty years of driving experience, has not known which steering feel model is used when the test started. Additionally, the steering feel model has been randomly selected by the simulation program for each test.

In all tests, the vehicle has started its motion from a stationary state and has been accelerated by the cruise control system according to the reference velocity. The data of interest presented as simulation results have been taken from the instant at which the vehicle enters into the test region. It has not been possible for the vehicle to enter the test region at the same instant for all tests. To be able to present neat and understandable plots, all test data have been shifted in time relative to a selected test data. For this purpose, crosscorrelation technique has been employed to find the lag between data by measuring their similarity. Therefore, the reader should note that some plots have been shifted in time.

4.1. Weave Test. According to ISO 13674 [44], the open-loop test procedure requires the steering wheel to be subjected to an oscillatory input. The vehicle is driven at the test speed and a sinusoidal steering input is applied by a human driver. A similar test approach based on this standard test procedure has been used in this study.

In the graphical test environment, a test track following a straight line path has been created and pylons at $40 \mathrm{~m}$ intervals were placed. The speed of vehicle has been kept constant during the tests through a cruise controller. A human driver has tried to keep the angular velocity of steering wheel as near constant as possible during the tests.

In the weave tests, both the hysteresis-based model and SAT-based model have been used to generate the steering wheel torque feedback. In Figures 8 and 9, the results obtained through the use of SAT- and hysteresis-based models in the tests are given. The longitudinal velocity of the vehicle has been kept near $60 \mathrm{~km} / \mathrm{h}$. The frequency of the sinusoidal inputs applied by the driver is around $0.2 \mathrm{~Hz}$. In the five weave tests, for each steering feel model, the driver has tried to drive around 25 pylons. In order to show that both models are matched, a result of weave tests completed with both models is chosen. The torques produced in the steering wheel through both models during the weave test are shown in 


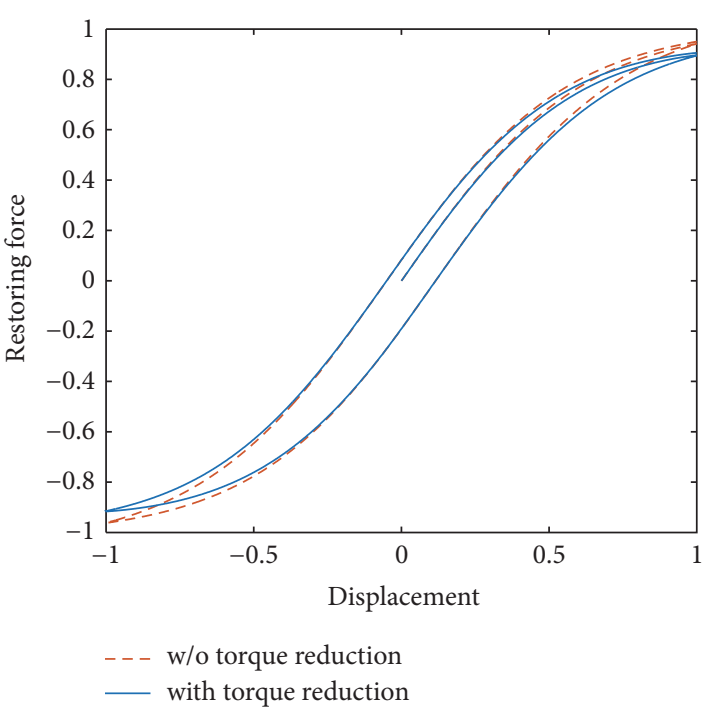

(a)

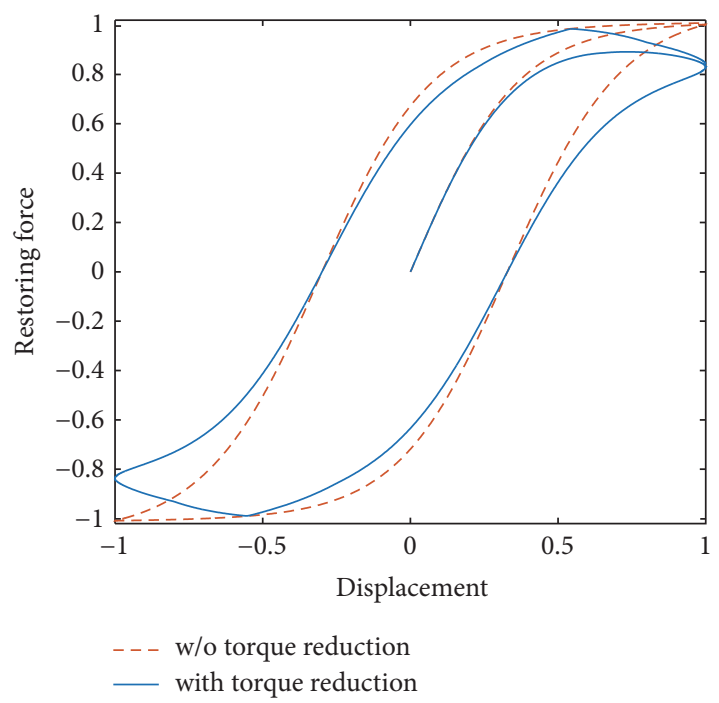

(c)

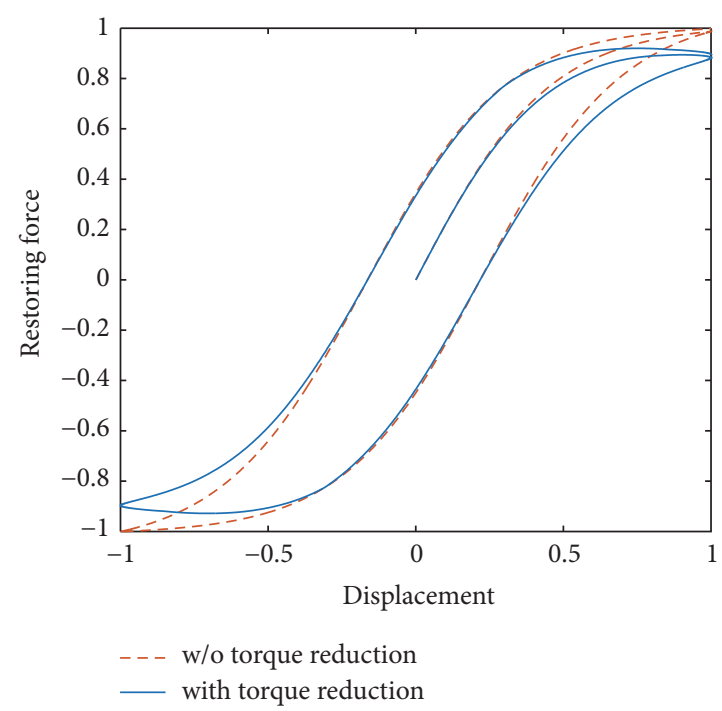

(b)

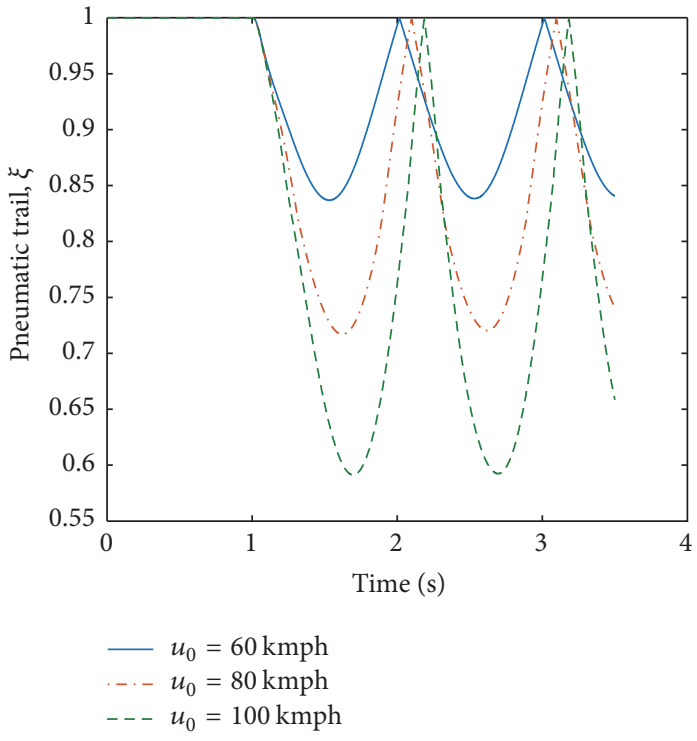

(d) Time history of pneumatic trail

Figure 6: $\Phi(x)(t)$ with and without torque reduction for (a) $u_{0}=60 \mathrm{kmph}$, (b) $u_{0}=80 \mathrm{kmph}$, and (c) $u_{0}=100 \mathrm{kmph}$.

Figure 8. The data are not shifted in time to be able to present both signals clearly. This plot indicates that the peak torque magnitudes are within the same band, and the overall magnitudes can be used to calculate the workloads.

The time histories of lateral displacements enable us to decide whether a comparison can be made between two sets of tests. In Figure 9(a), averages of lateral displacements obtained from each five weave tests are shown. The plots make clear that the vehicles have followed very similar paths around the pylons. Since the deviations of lateral displacements with respect to each other are quite small as seen in Figure 9(a), it is understood that the steering motions have been very similar and, thus, a fair comparison between the tests can be made. By looking at the lateral acceleration of the vehicles, Figure 9(b), almost the same responses for both cases can be identified.
The steering wheel angle versus steering wheel torque plots are shown in Figure 9(c) for each set of tests. Even these plots seem quite similar; there are two main differences between the shapes in these figures: (i) the gradient in the hysteresis-based model is less than that of the SATbased model and (ii) the peak values in the SAT-based model are higher than that of the hysteresis-based model. Such difference has been made intentionally by designing the hysteresis loop with selection of parameters as $\kappa_{x}=$ $0.01, \kappa_{\omega}=1, \sigma=0.1, n=1$, and $k_{u}=0.1$. Having a smaller gradient means that the steering wheel becomes less stiff for the driver.

The effect of different steering feel models on the driver can be evaluated in the sense of physical workload, which describes the physical work done by the driver in turning the 


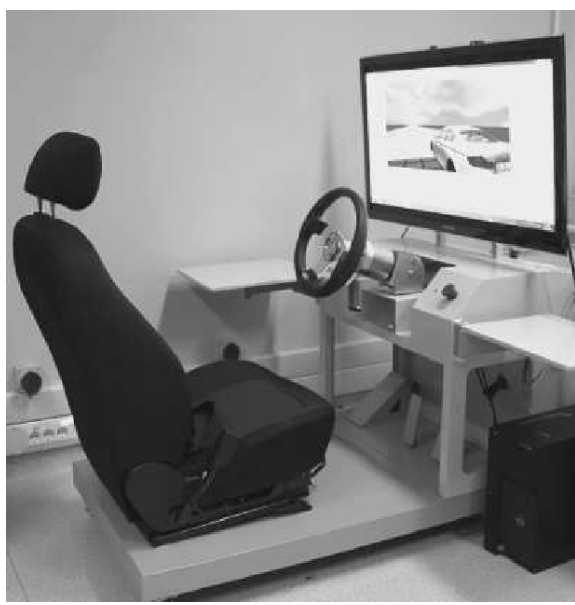

(a) Driving simulator

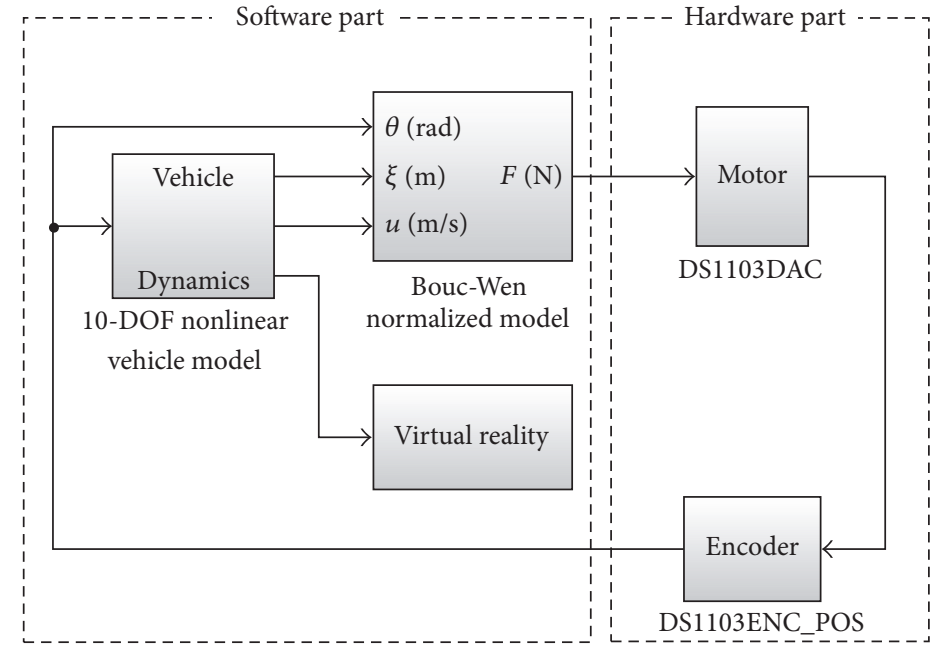

(b) General architecture of the HIL system

Figure 7: HIL simulation environment.

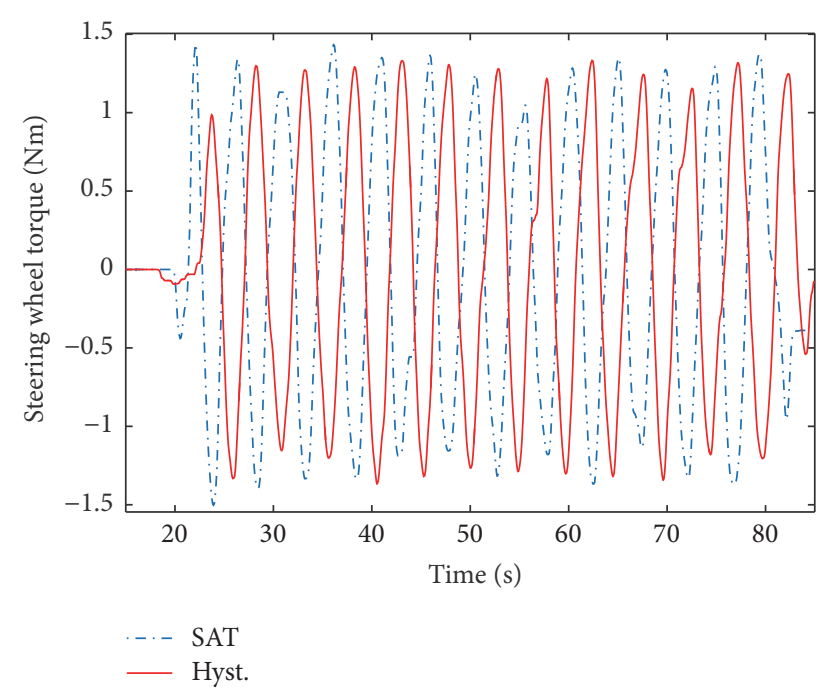

FIGURE 8: The torques produced in the steering wheel through both models during the weave test.

steering wheel. There are a few measures in the literature to describe the physical workload. In the work of Tajima et al. [45], the magnitude of physical workload is calculated by the integral of squared steering wheel angle rate. For this study, a new measure, the integral of steering power, is introduced in order to incorporate the steering wheel torque into the calculation of workload:

$$
J_{w}=\int_{0}^{t_{f}}\left|\dot{\theta}(t) T_{\mathrm{sw}}(t)\right| d t,
$$

where $t_{f}$ represents the final time for the steering task. Based on this measure, the workload has been calculated for each test, and the results are shown in Figure 9(d). The difference between the average values results from the difference between peak values seen in Figure 9(c).
All results of this test indicate that the given slalom task can be accomplished by the vehicle with both steering feel models. While the time histories of lateral acceleration given in Figure 9(b) are very close to each other, the physical workload values are different, Figure 9(d). Note that the smaller values of workload in the results of test of hysteresisbased model are due to the design of hysteresis shape as explained above. The significant result to be emphasized is that the same task has been accomplished with less effort of the driver in the vehicle with hysteresis-based model. By setting the parameters for the hysteresis-based model to have smaller gradient than the SAT-based model, the driver's workload has been decreased.

4.2. Double Lane-Change Test. To test the performance of hysteresis-based model in a severe lane-change manoeuvre, a test scenario has been created based on the standard ISO 3888-1 [46]. Compared to the weave test, this test is used to investigate the transient road-holding abilities of a vehicle. A vehicle at a given speed is first driven to another parallel lane and then returned to the initial lane, without exceeding lane boundaries. The entry speed has been selected as $60 \mathrm{~km} / \mathrm{h}$, and the double lane-change track has been designed based on the dimensions given in the standard ISO 3888-1. The results for this test are given in Figures 10(a)-10(d).

In Figure 10(a), the averages of lateral displacements obtained from each five weave tests are given for the same reason discussed in Section 4.1. The plots make clear that the vehicles have followed very similar paths around the pylons. Since the deviations of lateral displacements with respect to each other are quite few as seen in Figure 10(a), it is understood that the steering motions have been very similar and, thus, a fair comparison between the tests can be made. By looking at the lateral acceleration of the vehicles, Figure 10(b), almost the same responses for both cases can be identified.

The steering wheel angle versus steering wheel torque plots are shown in Figure 10(c) for each set of tests. The 


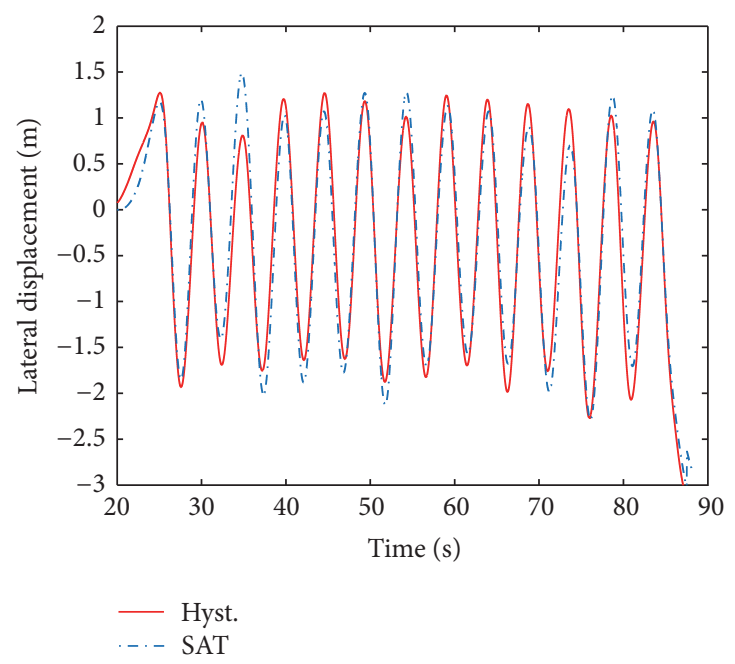

(a) Average lateral displacements of the vehicles

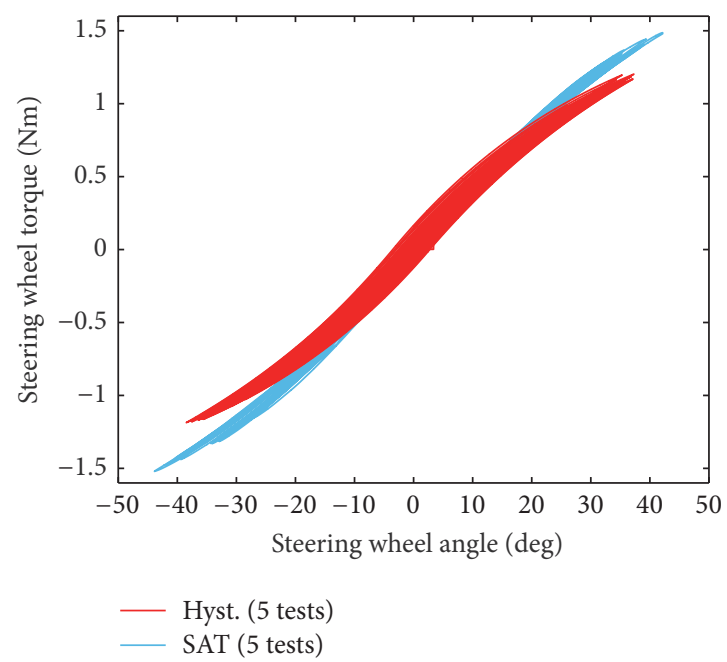

(c) Steering wheel torque generated by the models

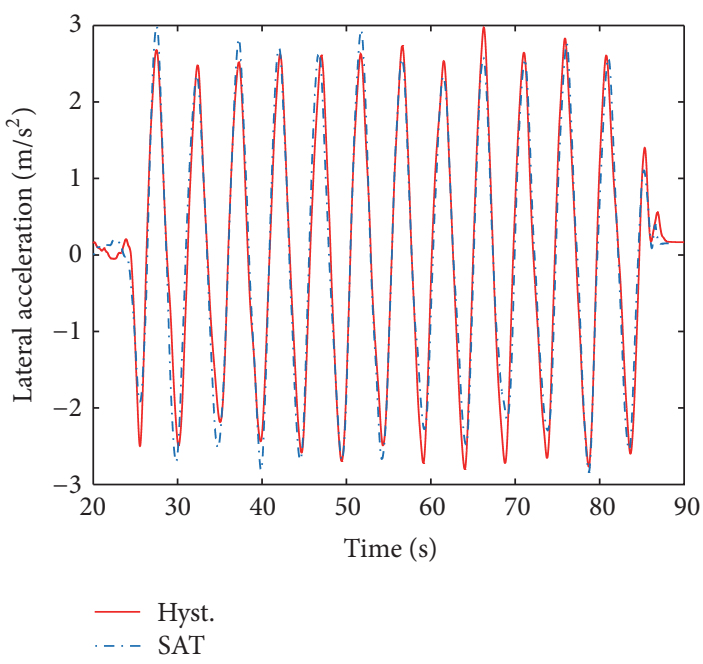

(b) Average lateral acceleration of the vehicles

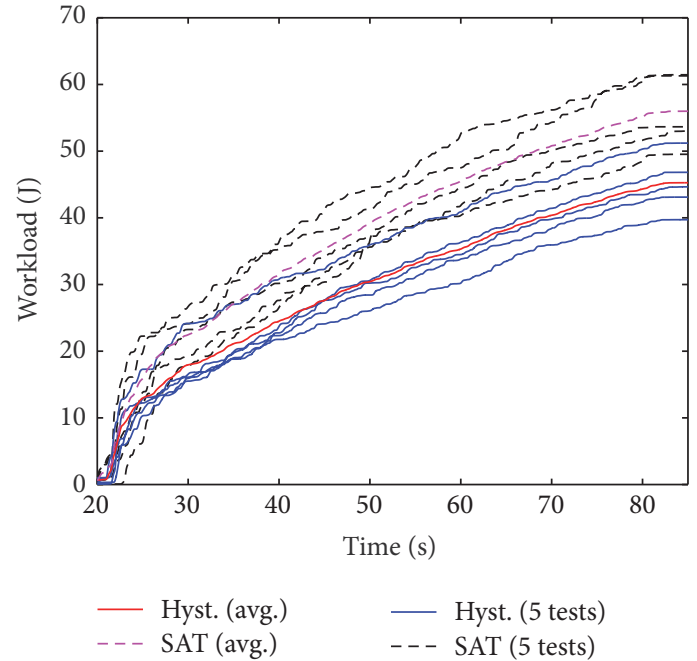

(d) Driver's physical workload

FIGURE 9: Weave test results.

same parameters given in Section 4.1 for the hysteresis-based model have been used for this case as well. The generated steering wheel torque is so affected by the rapid lateral movement of the vehicle that the upper and lower parts of the shapes seen in Figure 10(c) become curved compared to the ones in Figure 9(c). The change of lateral tire forces as a consequence of higher tire slip angles is responsible for such differentiation.

Based on the measure given in (10), the workload has been calculated for each test, and the results are shown in Figure 10(d). In this case, the difference between peak values seen in Figure 10(c) does not explain the difference between the both average values of physical workloads. The reason is that lower steering wheel angles and velocities have been experienced by using the hysteresis-based model.

\section{Conclusion}

The design of a steering feel by using a hysteresis model was proposed and its tests were studied in this paper. The motivation behind using a hysteresis model is that the hysteresis-based steering feel model can pattern the hysteresis characteristic appearing in the conventional steering systems. The mathematical model of the steering feel is based on the Bouc-Wen model of hysteresis. The model was modified for designing the steering feel, and optional parameters were added to facilitate the adjustment of hysteresis shape. While incorporation of the tire road interaction to the model and its speed dependency make the steering feel model more realistic, the availability of hysteresis shape parameters of the Bouc-Wen model makes it a flexible model.

To evaluate the hysteresis-based model, an HIL simulation environment was developed. The steering wheel system as the hardware-under-test was interfaced with the software part by a controller board, in which the steering feel model and vehicle simulation model were embedded. The vehicle model used in the experiment is a 10-DOF full vehicle model. The steering feel model was tested based on standard test approaches using the HIL system for two case studies; 


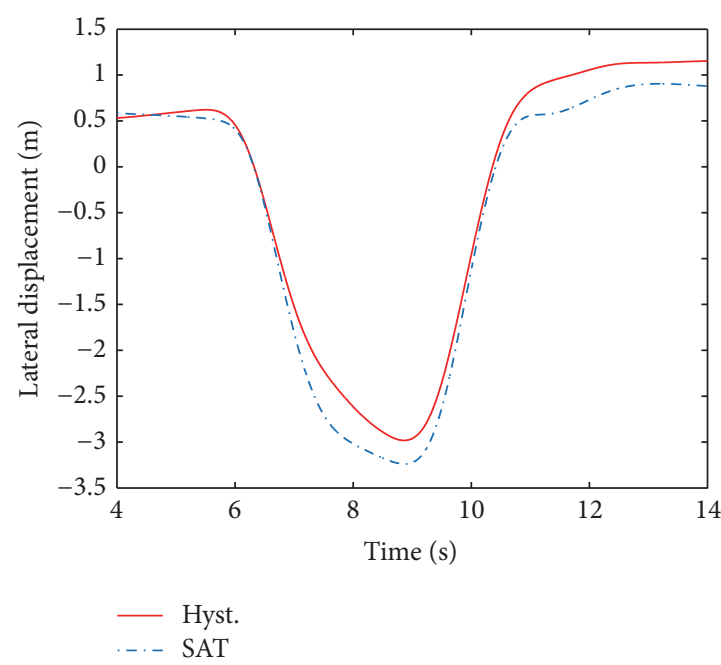

(a) Average lateral displacements of the vehicles

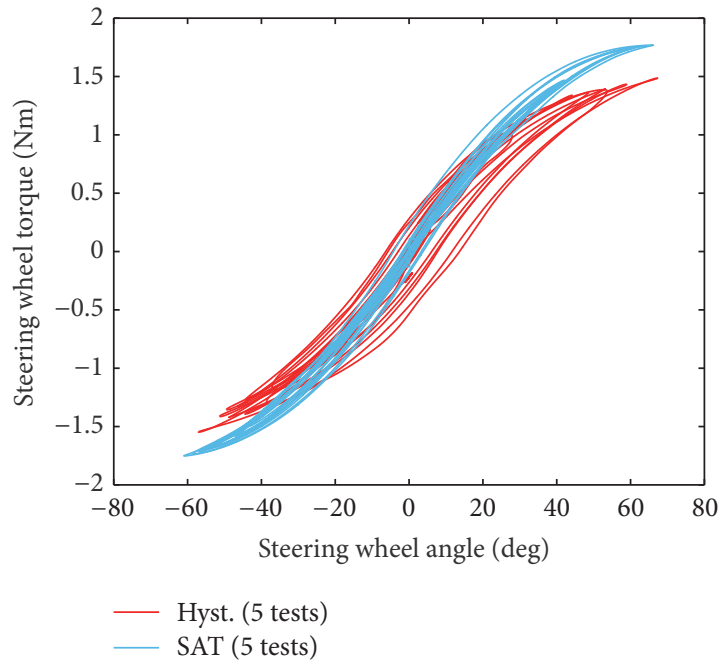

(c) Steering wheel torque generated by the models

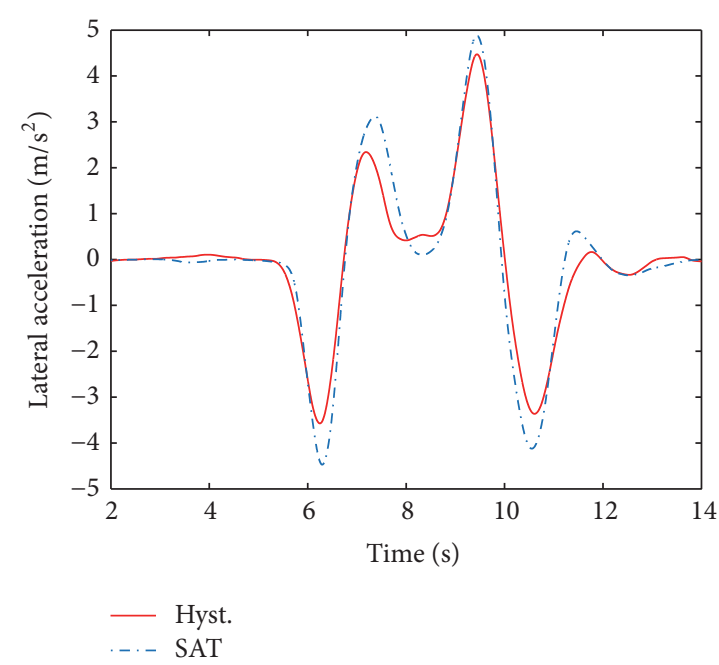

(b) Average lateral acceleration of the vehicles

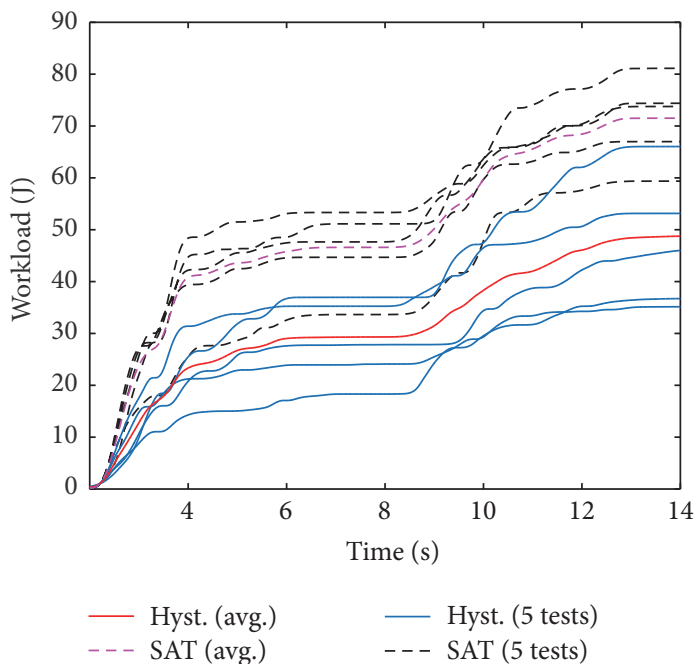

(d) Driver's physical workload

Figure 10: Double lane change test results.

the weave test and double lane-change test. The torque in the steering wheel motor was generated by either two models: hysteresis-based model and SAT-based model. In a comparative study, the performances of models were tested. In the weave test, the effect of steering feel on the dynamics of a vehicle was discussed by comparing the test results of both models. The same models were also tested for the double lanechange.

The results of tests have shown the effectiveness of the proposed steering feel model. The hysteresis-based model can be designed more easily compared to other models due to its design flexibility in wider ranges and forms. Since the steering related motion of road wheels or their interaction with the road is reflected to the model indirectly, even though the pneumatic trail is used in the model, this proposed model is not directly affected by the interference of active steering control commands. In conclusion, the research shows that hysteresis-based steering feel model provides a realistic and informative steering feel to enable a comfortable and safe driving, and the model would be a useful tool by providing the flexibility and the ease of design and tuning.

\section{Conflicts of Interest}

The author declares that there are no conflicts of interest regarding the publication of this paper.

\section{Acknowledgments}

This research is principally funded by The European Union, Marie Curie Actions-Support for training and career development of researcher [Project ID: PCIG09-GA-2011-294231, SteeringFeelSBW]. 


\section{References}

[1] L. Segel, "An Investigation of Automobile Handling as Implemented by a Variable-Steering Automobile," Human Factors: The Journal of Human Factors and Ergonomics Society, vol. 6, no. 4, pp. 333-341, 1964.

[2] M. K. Salaani, G. J. Heydinger, and P. A. Grygier, "Experimental steering feel performance measures," SAE Technical Papers, 2004.

[3] J. Black, P. T. Freeman, J. R. Wagner, E. Iyasere, D. M. Dawson, and F. S. Switzer, "Evaluation of driver steering preferences using an automotive simulator," International Journal of Vehicle Design, vol. 66, no. 2, pp. 124-142, 2014.

[4] M. Harrer, P. Pfeffer, and D. Johnston, "Steering Feel-Objective Assessment of Passenger Cars-Analysis of Steering Feel and Vehicle Handling," in Proceedings of the FISITA 2006 World Automotive Congress, Yokohama, Japan, 2006.

[5] D. Katzourakis, J. C. F. De Winter, S. De Groot, and R. Happee, "Driving simulator parameterization using double-lane change steering metrics as recorded on five modern cars," Simulation Modelling Practice and Theory, vol. 26, pp. 96-112, 2012.

[6] A. C. Newberry, M. J. Griffin, and M. Dowson, "Driver perception of steering feel," Proceedings of the Institution of Mechanical Engineers, Part D: Journal of Automobile Engineering, vol. 221, no. 4, pp. 405-415, 2007.

[7] J. Dang, H. Chen, B. Gao et al., "Optimal Design of On-Center Steering Force Characteristic Based on Correlations between Subjective and Objective Evaluations," SAE International Journal of Passenger Cars-Mechanical Systems, vol. 7, no. 3, 2014.

[8] A. K. Zschocke and A. Albers, "Links between subjective and objective evaluations regarding the steering character of automobiles," International Journal of Automotive Technology, vol. 9, no. 4, pp. 473-481, 2008.

[9] M. S. Arslan and N. Fukushima, "Energy optimal control design for steer-by-wire systems and hardware-in-the-loop simulation evaluation," Journal of Dynamic Systems, Measurement, and Control, vol. 137, no. 7, Article ID 071005, 2015.

[10] N. Fukushima, S. Ota, K. Hashimoto, M. S. Arslan, and I. Hagiwara, "A new vehicle control method based on tire dissipation power," in Proceedings of the 21st IAVSD Symposium on Dynamics of Vehicles on Roads and Tracks, pp. 17-21, Stockholm, Sweden, 2009.

[11] R. Pastorino, M. A. Naya, J. A. Pérez, and J. Cuadrado, “Geared PM coreless motor modelling for driver's force feedback in steer-by-wire systems," Mechatronics, vol. 21, no. 6, pp. 10431054, 2011.

[12] M. Segawa, "Vehicle stability control strategy for steer by wire system," JSAE Review, vol. 22, no. 4, pp. 383-388.

[13] A. E. Cetin, M. A. Adli, D. E. Barkana, and H. Kucuk, "Implementation and development of an adaptive steering-control system," IEEE Transactions on Vehicular Technology, vol. 59, no. 1, pp. 75-83, 2010.

[14] S. Asai, H. Kuroyanagi, S. Takeuchi, T. Takahashi, and S. Ogawa, "Development of a steer-by-wire system with force feedback using a disturbance observer," SAE Technical Papers, 2004.

[15] B.-H. Nguyen and J.-H. Ryu, "Direct current measurement based steer-by-wire systems for realistic driving feeling," in Proceedings of the International Symposium on Industrial Electronics (ISIE '09), pp. 1023-1028, Seoul, Republic of Korea, July 2009.

[16] C. J. Kim, J. H. Jang, S. K. Oh, J. Y. Lee, and J. K. Hedrick, "Development of a control algorithm for a rack-actuating steerby-wire system using road information feedback," Proceedings of the Institution of Mechanical Engineers, Part D: Journal of Automobile Engineering, vol. 222, no. 9, pp. 1559-1571, 2008.

[17] S. Amberkare, F. Bolourchi, J. Demerly, and S. Millsap, "A control system methodology for steer by wire systems," SAE Technical Papers 2004-01-1106, 2004.

[18] P. Setlur, D. Dawson, J. Chen, and J. Wagner, "A nonlinear tracking controller for a haptic interface steer-by-wire systems," in Proceedings of the 41st IEEE Conference on Decision and Control, pp. 3112-3117, December 2002.

[19] Driver Model Based Vehicle Handling Quality Evaluation-Effect of steering reaction torque on the evaluation, IAVSD09, 2009.

[20] S. W. Oh, H. C. Chae, S. C. Yun, and C. S. Han, "The design of a controller for the steer-by-wire system," JSME International Journal Series C Mechanical Systems, Machine Elements and Manufacturing, vol. 47, no. 3, pp. 896-907, 2004.

[21] M. K. Salaani, G. Heydinger, and P. Grygier, "Modeling and implementation of steering system feedback for the national advanced driving simulator," SAE Technical Papers, 2002.

[22] Y. H. J. Hsu and J. C. Gerdes, "A feel for the road: A method to estimate tire parameters using steering torque," in Proceedings of the International Symposium on Advanced Vehicle Control (AVEC), Taipei, Taiwan, 2006.

[23] A. Balachandran and J. C. Gerdes, "Designing Steering Feel for Steer-by-Wire Vehicles Using Objective Measures," IEEE/ASME Transactions on Mechatronics, 2014.

[24] H. Shimomura, T. Haraguchi, Y. Satoh, and R. Saitoh, "Simulation analysis on the influence of vehicle specifications upon steering characteristics," International Journal of Vehicle Design, vol. 12, no. 2, pp. 197-207, 1991.

[25] I. Yamazaki, I. Kushiro, and Y. Kunihiro, "Electronic power steering compensating control for influence of vehicle dynamics on steering torque," SAE International Journal of Passenger Cars-Mechanical Systems, vol. 2, no. 1, pp. 239-246, 2009.

[26] Y. Morita, K. Torii, N. Tsuchida et al., "Improvement of steering feel of Electric Power Steering System with Variable Gear Transmission System using decoupling control," in Proceedings of the 10th International Workshop on Advanced Motion Control, AMC'08, pp. 417-422, March 2008.

[27] R. W. Allen, T. J. Rosenthal, and H. T. Szostak, "Steady state and transient analysis of ground vehicle handling," SAE Technical Papers, 1987.

[28] G. J. Heydinger, W. R. Garrott, J. P. Chrstos, and D. A. Guenther, "Dynamic effects of tire lag on simulation yaw rate predictions," Journal of Dynamic Systems, Measurement, and Control, vol. 116, no. 2, pp. 249-256, 1994.

[29] A. Kirli and M. S. Arslan, "Online optimized hysteresis-based steering feel model for steer-by-wire systems," Advances in Mechanical Engineering, vol. 8, no. 7, pp. 1-15, 2016.

[30] M. Ismail, F. Ikhouane, and J. Rodellar, "The hysteresis BoucWen model, a survey," Archives of Computational Methods in Engineering: State-of-the-Art Reviews, vol. 16, no. 2, pp. 161-188, 2009.

[31] A. Visintin, Differential models of hysteresis, vol. 1, SpringerVerlag, Berlin, Germany, 1994.

[32] L. O. Chua and K. A. Stromsmoe, "Mathematical model for dynamic hysteresis loops," International Journal of Engineering Science, vol. 9, no. 5, pp. 435-450, 1971.

[33] D. C. Jiles and D. L. Atherton, "Theory of ferromagnetic hysteresis," Journal of Magnetism and Magnetic Materials, vol. 61, no. 1-2, pp. 48-60, 1986. 
[34] I. D. Mayergoyz, "Mathematical models of hysteresis (Invited)," IEEE Transactions on Magnetics, vol. 22, no. 5, pp. 603-608, 1986.

[35] F. Ikhouane and J. Rodellar, Systems with hysteresis: analysis, identification and control using the Bouc-Wen model, John Wiley and Sons, 2007.

[36] R. Bouc, "Modèle mathématique d'hystérésis," Acustica, vol. 24, no. 3, pp. 16-25, 1971.

[37] Y. K. Wen, "Method for random vibration of hysteretic systems," Journal of the Engineering Mechanics Division, vol. 102, no. 2, pp. 249-263, 1976.

[38] F. Ma, H. Zhang, A. Bockstedte, G. C. Foliente, and P. Paevere, "Parameter analysis of the differential model of hysteresis," Journal of Applied Mechanics, vol. 71, no. 3, pp. 342-349, 2004.

[39] R. Rajamani, Vehicle Dynamics and Control, Springer, 2011.

[40] Y.-H. Judy Hsu, S. M. Laws, and J. C. Gerdes, "Estimation of tire slip angle and friction limits using steering torque," IEEE Transactions on Control Systems Technology, vol. 18, no. 4, pp. 896-907, 2010.

[41] M. Abe, Vehicle Handling Dynamics Theory and Application, Butterworth-Heinemman, 2009.

[42] U. Kiencke and L. Nielsen, Automotive Control Systems for Engine, Driveline, and Vehicle, Springer Verlag, New York, NY, USA, 2nd edition, 2005.

[43] H. B. Pacejka Elsevier, 2005.

[44] "Road vehicles - Test method for the quantification of on-centre handling -Part 1: Weave test," ISO 13674-1:2010, 2010.

[45] J. Tajima, N. Yuhara, S. Sano, and S. Takimoto, "Effects of steering system characteristics on control performance from the viewpoint of steer-by-wire system design," SAE Technical Papers, 1999.

[46] "Passenger cars - Test track for a severe lane-change manoeuvre Part 1: Double-lane change," ISO 3888-1:1999, 1999. 


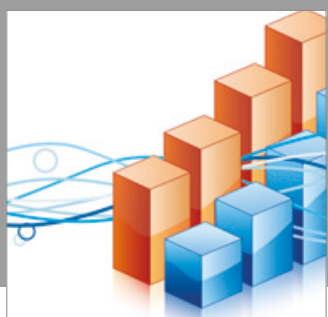

Advances in

Operations Research

vatersals

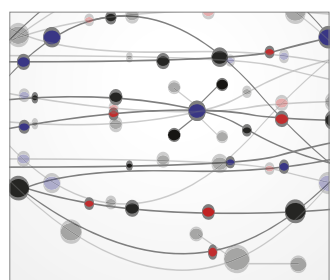

\section{The Scientific} World Journal
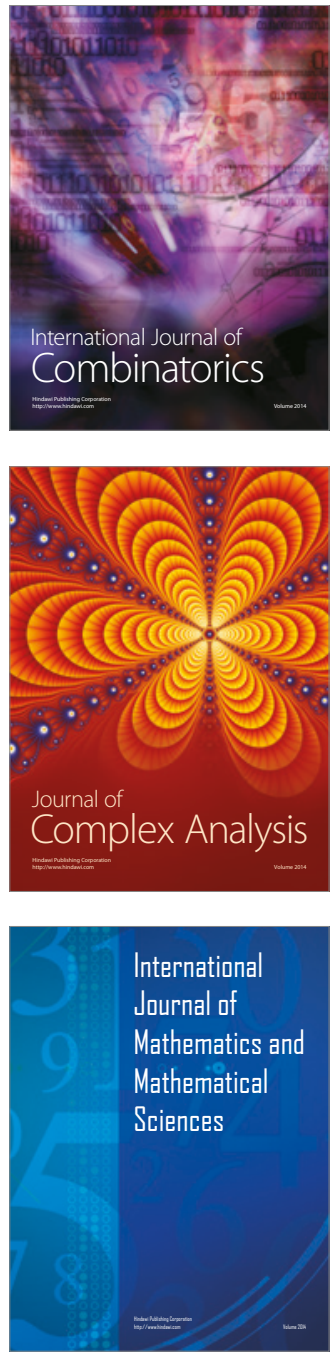
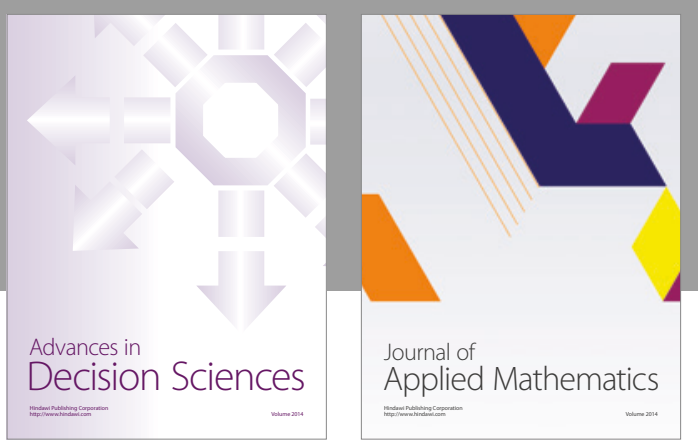

Algebra

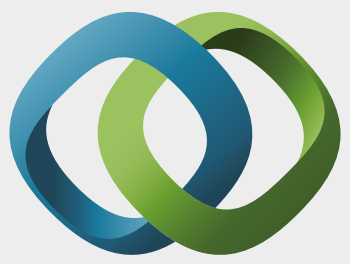

\section{Hindawi}

Submit your manuscripts at

https://www.hindawi.com
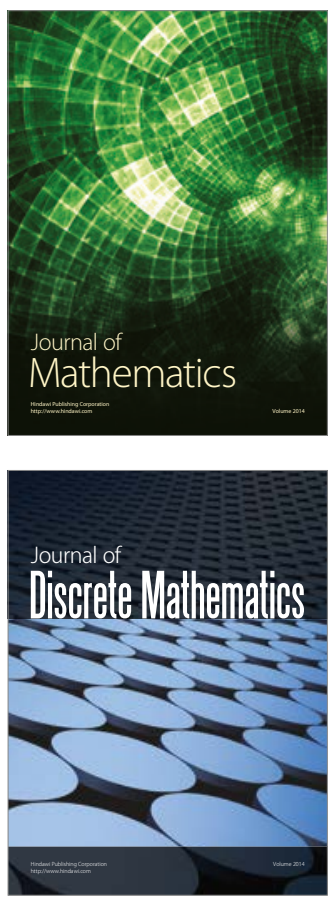

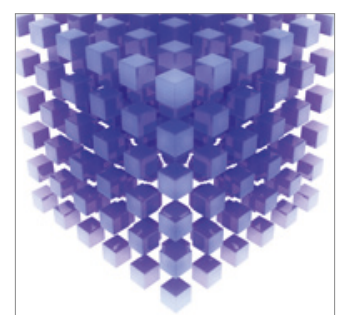

Mathematical Problems in Engineering
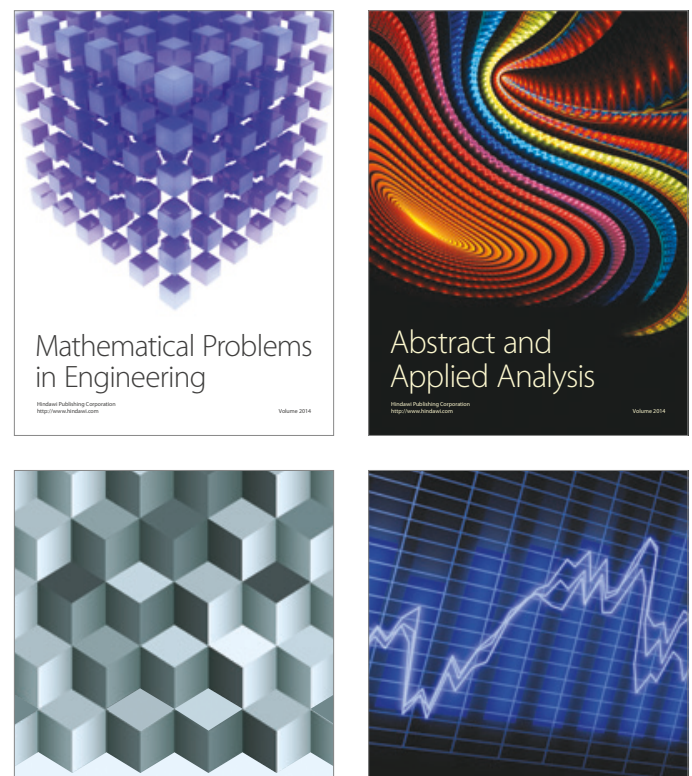

Journal of

Function Spaces

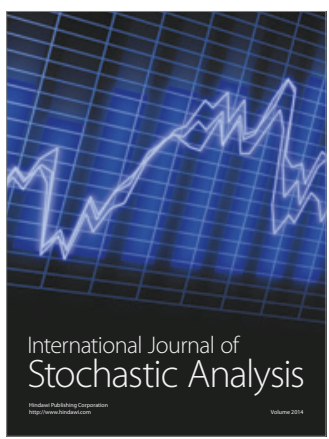

Probability and Statistics
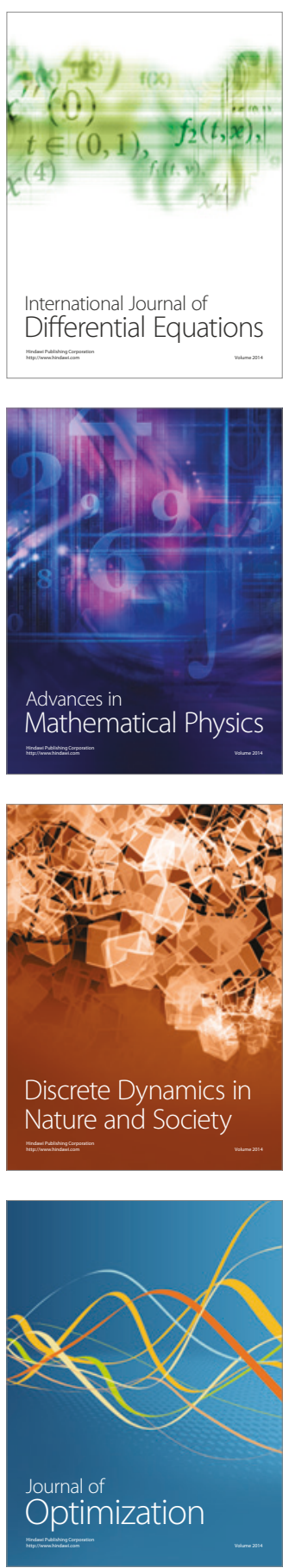